\title{
Integrated Voltage and Power Flow Management Considering the Cost of Opera in Active Distribution Networks
}

\author{
Tao Xu*, Lingxu Guo**, Wei Wei ${ }^{\dagger}$, Xiaoxue Wang*, Chengshan Wang*, Jun Lin*** \\ and Tianchu $\mathrm{Li}^{* * *}$
}

\begin{abstract}
The increasing penetration of distributed energy resources on the distribution networks have brought a number of technical impacts where voltage and thermal variations have been identified as the dominant effects. Active network management in distribution networks aims to integrate distributed energy resources with flexible network management so that distributed energy resources are organized to make better use of existing capacity and infrastructure. This paper propose active solutions which aims to solve the voltage and thermal issues in a distributed manner utilizing a collaborative approach. The proposed algorithms have been fully tested on a distribution network with distributed generation units.
\end{abstract}

Keywords: Active distribution networkk, Voltage control, Power flow management, Distributed energy resource, Multiple agent system

\section{Introduction}

The rapid growth of distributed generation (DG) connections have promoted the proportion of electricity generation from renewable sources at all voltage levels. The stochastic nature of the power output of most forms of DG units result in a number of planning and operational uncertainties to the distribution network operators (DNOs) including voltage variations, bidirectional power flow, and system perturbations like harmonics or flickers; voltage variations and power flow uncertainties have been identified as the dominant effects [1]

- Intermittent power injections and unpredictable load may cause voltage excursions on weak distribution lines under extreme generation and loading conditions.

- Reverse power flows from DG units under low load and high generation scenarios may breach the thermal ratings on lines and transformers.

The existing 'fit-and-forget' approach to connecting DG to a network requires adequate capacity for DG export at will under all circumstances and therefore requires expensive reinforcement as the DG penetration grows [2] The overall passive scheme and cost burden of reinforcement are making potential DG connection less attractive and, in some instances, jeopardize the development of the renewable energy project.

$\dagger \quad$ Corresponding Author: Key Laboratory of Smart Grid of Ministry of Education, Tianjin University, China. (weiw@tju.edu.cn)

* Key Laboratory of Smart Grid of Ministry of Education, Tianjin University, China. (taoxu2011@tju.edu.cn)

** Tianjin Electric Power Co., China. (lingxuguo@126.com)

*** Hainan Electric Power Research Institute, China

Received: April 23, 2015; Accepted: September 23, 2015
Consequently, under existing technical and commercial arrangements, utilities are eager to seek solutions which can allocate the network latent capacity to new connections and demands to the grid while avoiding, reducing or deferring expensive network reinforcement costs. Active network management (ANM) solutions make it possible. ANM solutions monitor and control the grid in real time to integrate DG operation with network management so that DG are controlled to make better use of existing capacity and infrastructure [3] ANM forms a new layer in grid management systems, sitting between the supervisory control and data acquisition (SCADA) systems and the protection, automation and control devices deployed to substations [4]

The ANM scheme involves intelligent measurement, stable communications and active control schemes within the network. If problems arise in the network ANM schemes can make decisions upon the electrical assets to improve network operation, such as making decisions on DG operations, to mitigate their operational problems. This could allow for the increase in DG connections to continue within distribution networks, without the need for costly reinforcement as well as reducing connection expenses [5] Recent studies have demonstrated that ANM schemes are capable to create up to two or three times more capacity to host new generation and demand [6]

The UK Orkney islands ANM project has been implemented to allow generators to export more power to the grid, thermal constraint management as a single operational objective rather than the full state of the network has been managed in an active way, this has allowed for a reduction in communication and the complexity of solutions, allowing for the autonomous scheme to run smoothly and securely in a timely fashion [7] 
Collins etc. has addressed the voltage regulation issues utilizing a fully decentralized ANM system. It employs a multiple agent system (MAS) that aims to maintain the voltage inequality constraints whilst minimizing the cost of corrective actions [8]

In fact, most relevant research activities carried out were concentrated on an individual aspect of network issues [9-12] From a grid perspective, the voltage and power flow are tightly coupled matters which can be considered in a collaborative approach. The overall grid restrictive constraint can either be voltage limit or thermal threshold depends on the types of network, natures of DG and its ways of connection. Studies have shown that voltage limit represent the major constraints to DG penetration in rural networks, while thermal limits are the most restrictive factor in urban networks in case of direct connection to the feeders [13]

This paper proposed an ANM scheme that integrate voltage and power flow management (PFM) solutions, a bespoke self-adaptive similarity assessment approach and an on-line constrained satisfaction (CSP) algorithm are implemented to solve the voltage and power flow issues. An artificial operating cost approach is introduced to investigate the practicability and the feasibility with consideration of the nature of DG and the DNO's preference.

The paper is structured as follows: Section 1, introduces the background of the system and proposed system architecture. The voltage control (VC) and PFM schemes are discussed in Section 2 and Section 3 respectively. And Section 4 introduces the artificial operating cost approach and the collaborative management architecture is presented in Section 5. In Section 6 the closed-loop test environment and the test scenarios, as well as the testing results are presented. And the conclusions and discussions are drawn in Section 7.

\section{Voltage Control}

A voltage excursion is defined as a voltage magnitude change that results in an out-of-specification anywhere on a network operating in its normal state [1] In comparison to transmission lines, the typical $\mathrm{R} / \mathrm{X}$ ratio of a distribution network is relatively high; any significant power injection of DG units or heavy load conditions may result in voltage excursions on the distribution networks, especially in a weak distribution feeder with high impedance. Voltage excursions could occur under the following situations under normal operating condition:

- DG operating at maximum output during minimum load times;

- DG operating at low output, or disconnected from the network during peak load times;

Currently, the most common measure to maintain a

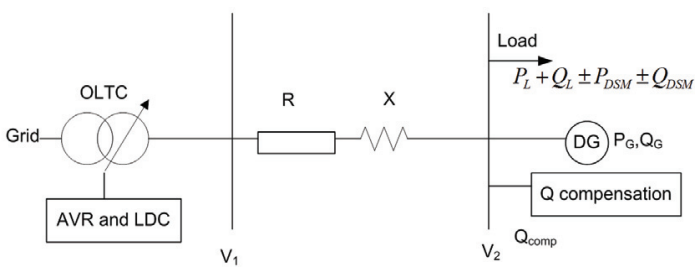

Fig. 1. Simple radial feeder with connected DG

stable secondary voltage employed on distribution networks is the use of on-load tap changers (OLTCs) [1] The OLTC usually operates in conjunction with an automatic voltage regulator (AVR) relay and line drop compensator (LDC) as shown in Fig. 1. The AVR relay continuously monitors the output voltage from the transformer, a tap change command will be initiated when the voltage is beyond the pre-set limits. The LDC is used to compensate for the voltage drop on the line between the transformer and loads situated toward the far end of the feeder.

A simplified distribution feeder with a DG connected is shown in Fig. 1. The voltage variation across the feeder can be approximated by the Eqs. (1-3):

$$
\Delta V=\frac{R P_{N E T}+X Q_{N E T}}{V}
$$

where

$$
\begin{gathered}
P_{N E T}=P_{G}-P_{L} \pm P_{D S M} \\
Q_{N E T}= \pm Q_{G}-Q_{L} \pm Q_{D S M} \pm Q_{c o m p}
\end{gathered}
$$

Where, $\Delta V$ indicates voltage variation across the feeder, $P_{G}$ and $Q_{G}$ represent the DG real and reactive power output, $P_{L}$ and $Q_{L}$ indicate the real and reactive power of the load; $P_{D S M}$ and $Q_{D S M}$ are the real and reactive power output of demand side management scheme; $Q_{\text {comp }}$ is the output of Q compensator; $X$ and $R$ are the reactance and resistance of the line between the substation and the DG, $V$ is the nominal voltage at the terminal of DG.

The increasing integration of distributed controllable resources on the network can add complexity and uncertainties to the voltage variations dramatically. The risks of non-convergence of online $\mathrm{VC}$ schemes, such as optimal power flow (OPF), can result in unsolved voltage rising issues. Consequently, a self-learning SQL-based approach to similarity assessment within a relational database has been proposed in this paper as a case base reasoning (CBR) approach for $\mathrm{VC}$ solution. It is not only useful in cases where a vast amount of experience can be drawn upon, but also when knowledge is incomplete [14]

The proposed self-learning CBR approach involves two phases of work: offline and online. The offline process focus on case base construction and the online process concentrates on the functionality of similarity assessment and self-learning of the case base. 


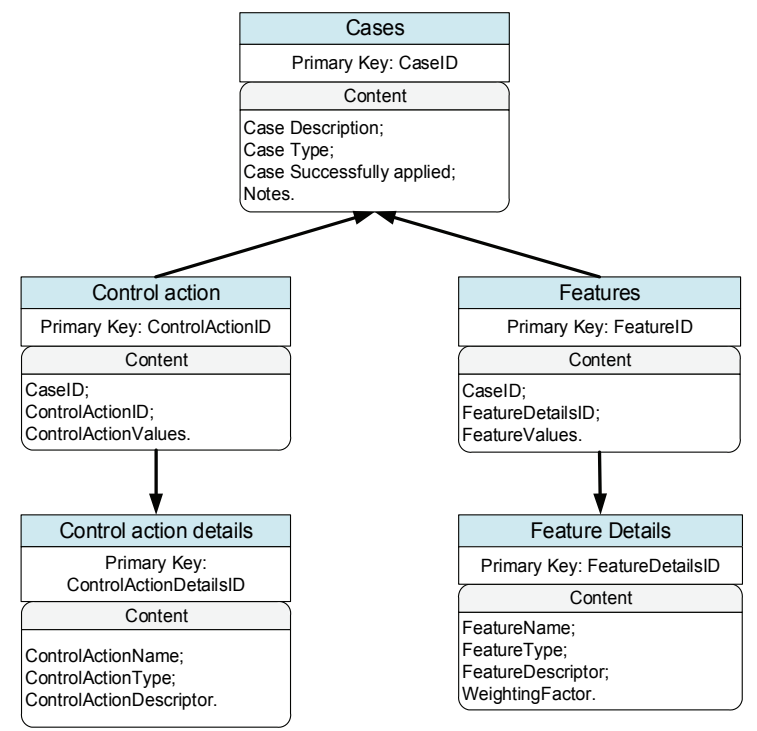

Fig. 2. Generic case base design

\subsection{Offline case base construction}

The rational database stores individual aspects of network information in a case base which allows fast construction of a knowledge base. The identified voltage excursion scenario is being wrapped as a case that is determined to be similar to the problem are retrieved with their corresponding solutions from the case base. Fig. 2 explains the design of the case base, the 'Feature details' is used to record the status of individual network equipment, for instance, the tap position of an OLTC with a corresponding feature details ID. The 'Featues' is constructed by extracting information from the 'Feature Details'. Similarly, the 'Control action' is also created utilizing information from 'Control action details'. Once the databases of 'Control action' and 'Features' are compiled, the database 'Cases' is therefore built with features and control actions, and the 'caseID' is utilized as the link between the features and the corresponding control actions.

The case base proposed in this paper has been constructed utilizing OPF with DG power output maximization as the target.

\subsection{Online case retrieval and self-learning}

Giving consideration to the features of distribution systems, a nearest neighbor (NN) retrieval technique along with CBR self-learning and feedback processes are adopted in this approach. The NN approach is implemented to retrieve the closest match for each case by comparing the case feature values. A similarity for each feature i can be calculated by Eq. (4):

$$
S_{f i}=1-\frac{\left|V_{c i}-V_{p i}\right|}{R}
$$

The overall similarity can be expressed as:

$$
S_{O}=\frac{\sum_{i=1}^{N} S_{f i} \times \omega_{f i}}{\sum_{i=1}^{N} \omega_{f i}}
$$

Where, $S_{f i}$ and $S_{O}$ represent the similarity for an individual feature $i$ and the overall similarity for a problem case respectively, $V_{c i}-V_{p i}$ is the difference between a feature case value and current value, $R$ is the range of an individual feature, $N$ is the total number of case features, and $\omega_{f i}$ indicates the weighting factor for each feature which is pre-defined in the case base from expert knowledge. According to the expert knowledge from the DNO and offline studies, higher weighting factors are given to the important features, such as, DG power output, tap position and voltage levels on busbars close to the DG connection points. For the tests described in this paper, a weighting of 1 is given to each of the important features and 0.9 is given to each of other features.

The retrieved cases are sent to the online verification process to testify the solution with altered network parameters. The successful verified solution with highest similarity is ready for implementation to the network to achieve the close loop control. Furthermore, the newly identified voltage excursion case with corresponding solutions are being added into the case base and the cases which failed in verification are revised or deleted based on the verification / implementation experiences. The selflearning process eases the case base maintenance and integrates with real operation. The VC software design is being illustrated in Fig. 3.

Compared to online VC methods, such as OPF, the proposed CBR approach is capable of managing different types and sizes of networks without risks of nonconvergence; and it is able to cope with incomplete information, for instance, data corruption and model errors; as well as to provide practical solutions which have been verified rather than deriving from first principle.

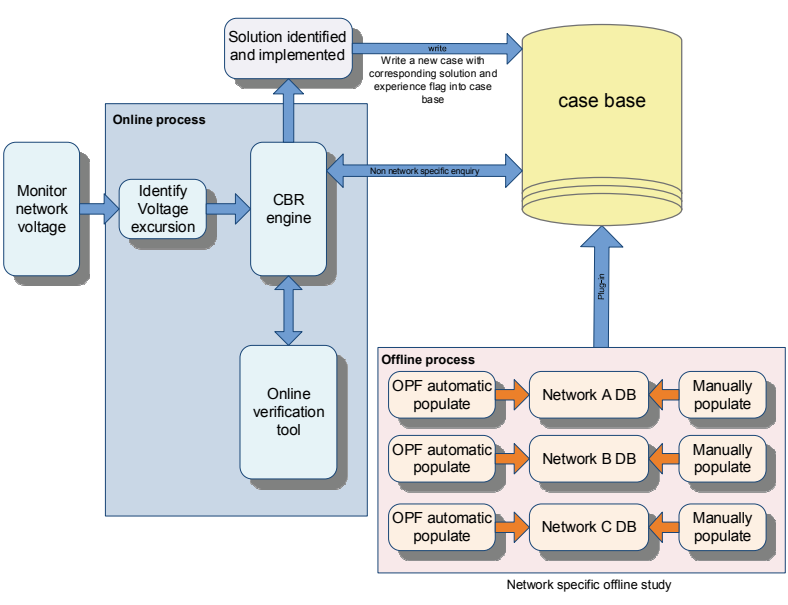

Fig. 3. Software design for voltage control function 


\section{Power Flow Management}

Thermal limits become one of the main barriers on the firm DG connections for a network without reinforcement. Therefore the DNOs are keen to employ active schemes which can offer a means connecting more DG units than the level allowed by traditional system planning [15]

PFM in active distribution network indicates the active management of controllable resources on the network such that the thermal limits of plant are not exceeded [16] Due to the nature of the active PFM scheme which involves multiple constraints, constraint satisfaction programming (CSP) has been chosen as the proposed solution. The CSP is defined as an objective function problem presented in a certain mathematical model that offer a means of applying constraints to the variables of a specific problem initially embedded within the logic programming field [17] The declarative programming approach includes triple factors: a set of variables with finite discrete domains and a set of constraints.

For active PFM problem applications, the CSP variables are controllable DG / loading power output set-points, each set-point has a domain of discrete values that the generators and the controllable loads may be set to, and a set of constraints such as power flow constraints and DG power output restrictions are involved. (9).

The proposed CSP algorithm is represented in Eqs. (6)-

$$
P_{C S P}=\left(X, D_{\text {control }}, C\right)
$$

Where $\mathrm{X}$ is the finite set of variables contains controllable generating/loading power output set-points:

$$
X=\left\{X_{D G 1}, X_{D G 2}, \ldots \ldots ., X_{D G n}, X_{\text {load } 1}, X_{\text {load } 2}, \ldots \ldots ., X_{\text {loadm }}\right\}
$$

$\mathrm{D}$ is the domain with optional discrete values that can be assigned to the controllable DG and load:

$$
\begin{aligned}
& D_{D G 1}=\{1,0\}, \\
& \mathrm{D}_{D G 2}=\left\{v_{1}, v_{2}, \ldots, \mathrm{v}_{n}\right\}, \\
& \mathrm{D}_{\text {load } 1}=\left\{u_{1}, u_{2}, \ldots, u_{n}\right\}, \ldots \ldots
\end{aligned}
$$

C is the constraints applied to the sets of variables, such as:

$$
\begin{aligned}
& C_{\text {powerflow }}=\left\{\left|S_{i j}\right| \leq \mathrm{S}_{i j}^{\max }\right\} \\
& C_{\max D G}=\left\{\max \sum_{i=1}^{N} P_{G i}\right\} \text { etc }
\end{aligned}
$$

\begin{tabular}{|c|c|}
\hline Number & Event \\
\hline 1 & $\begin{array}{l}\text { while not solved } \underline{\text { and }} \text { Check consistency } \\
\text { not infeasible } \underline{d o}\end{array}$ \\
\hline 2 & $\begin{array}{l}\text { if a dead end is detected } \\
\frac{\text { then }}{\text { try to escape from dead end }}\end{array}$ \\
\hline 3 & $\begin{array}{l}\frac{\text { else }}{\text { select variables }} \\
\text { select values for variables }\end{array}$ \\
\hline 4 & $\begin{array}{l}\text { endif } \\
\text { endwhile }\end{array}$ \\
\hline
\end{tabular}

Where $\mathrm{S}_{i j}$ and $\mathrm{S}_{i j}^{\max }$ represent the line power flows and associated limits separately, $N$ is the total number of DG units and $P_{G i}$ is the output of each DG. Table 1
Table 1. Framework of CSP search algorithms

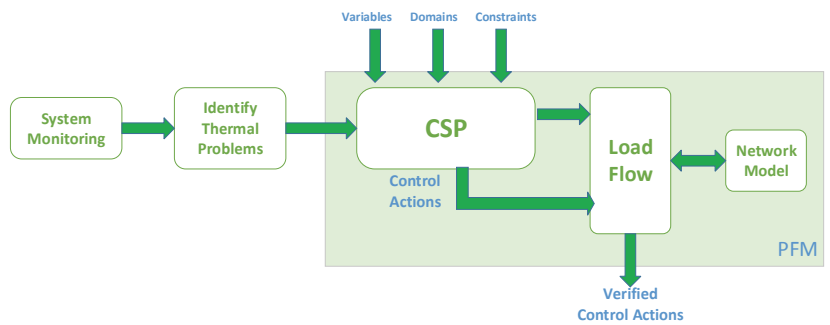

Fig. 4. Software design for PFM function

explains the procedures that the CSP algorithm search for feasible solutions within the bounds of PFM problem and the PFM system software design is illustrated in Fig.4.

\section{Artificial Operating Cost}

During the real network operation, the DNOs seek to find appropriate solutions not only having the capabilities to manage the voltage and thermal but leaving the system with better conditions and higher revenue. Therefore, artificial operating cost including cost of voltage and active/reactive power curtailment are introduced.

\subsection{Cost of voltage}

The CBR system retrieves $\mathrm{N}(\mathrm{N}$ is an integer pre-defined by the operators) closest matches with corresponding VC solutions ranked by similarities. And each of the solution is required to do the online verification which needs to run load flow with altered parameters. The load flow results show the voltage variation after control. Therefore, a voltage constraint algorithm which consider the artificial cost of operation has been adopted. The artificial voltage cost is based on a bath tub curve shown in Fig. 5 which is approximately zero when the voltage level is kept within predefined operating constraints, but becomes extremely large when the voltage level moves away. The mathematic model of the artificial voltage cost is illustrated in Eq. (10).

$$
C(V)=[C(1-V)]^{m}
$$

Where $C(V)$ represents the cost associated with 


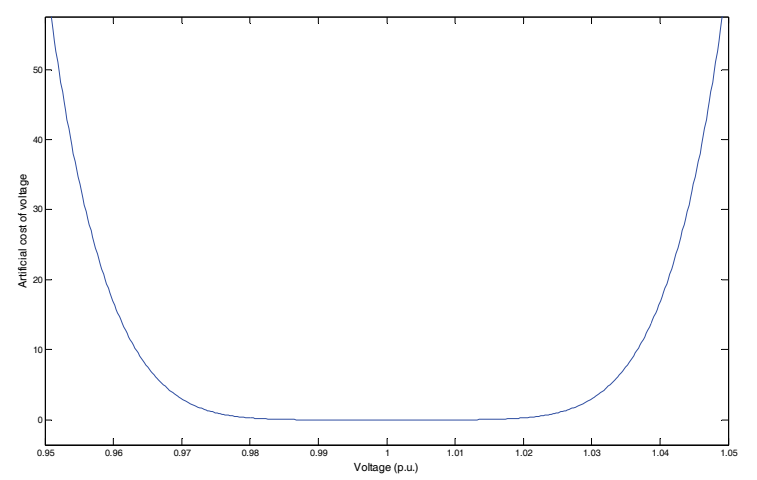

Fig. 5. Artificial cost of voltage

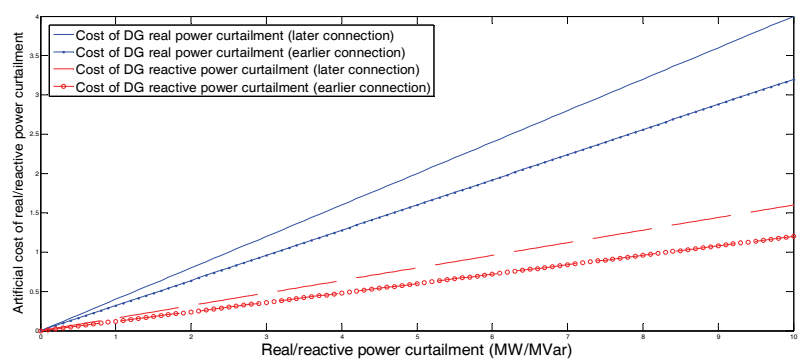

Fig. 6. Artificial cost of power curtailment with two DG

voltage, $\mathrm{C}$ and $\mathrm{m}$ are constants determined by the shape of the bathtub diagram expressed in Fig. 5. According to the analysis, $C=40, m=6$ are chosen in the case study for this paper.

\subsection{Cost of DG power curtailment}

In order to manage the DG power curtailment for constraint management, a generic cost solution refers to the cost of correcting DGs' active/reactive output with consideration of last-in-first-off (LIFO) policy is implemented. LIFO policy means the last DG units to connect on the system is the first to be curtailed. The artificial cost function is explained in Eq. (11) for details and Fig. 6 shows an example of two DG (with different year of connection) power curtailment and their associated cost.

$$
\begin{aligned}
& C\left(P_{G N}\right)=M_{P}\left(P_{G N \max }-P_{G N}\right) \\
& C\left(Q_{G N}\right)=M_{Q}\left(Q_{G N \max }-Q_{G N}\right)
\end{aligned}
$$

Where $C\left(P_{G N}\right)$ and $C\left(Q_{G N}\right)$ represent the cost of each constrained DG, $M_{P}$ and $M_{Q}$ are the cost of power per unit where $M_{P}>M_{Q}, P_{G N}$ and $Q_{G N}$ are the desired DG operational point, and $P_{G N \max }, Q_{G N \max }$ are the maximum operational power the $\mathrm{DG}$ can produce in regards to the available energy sources.

The overall system operating cost can be calculated by Eq. (12)

$$
C_{s y s t e m}=C(\mathrm{~V})+\sum_{i=1}^{N}\left[C\left(\mathrm{P}_{G N_{i}}\right)+\mathrm{C}\left(\mathrm{Q}_{G N_{i}}\right)\right]
$$

where $N$ represent the total number of DG units.

By integrating with the similarity score of individual retrieved case from $\mathrm{CBR}$, an overall score can be calculated by Eq. (13)

$$
\text { Score }=\left(S_{O}-0.5 \times C_{\text {system }}\right) \times v
$$

Where $v$ indicates the online verification result, if the solution pass the verification $v=1$, otherwise $v=0$.

\section{Collaborative Management}

The ANM approaches range from centralized to fully decentralize which requires different levels of measurements, control and communication infrastructures. Due to the communication and computation burdens, the centralized control architecture has always been adapted to hybrid control schemes including an arbitration agent which make final decision subject to the overall system objective. MASs are normally utilized as the platform to realize the ANM, each agent represents either a functionality or a specific cell on the system. Fig. 7 illustrates the proposed MAS platform to perform distributed decision making on the dispatch of DERs to control network voltages or thermal within prescribed limits. The arbitration function is utilized to make the final decision to avoid agent confliction.

In this paper, as the voltage and thermal are organized through two independent intelligent approaches: CBR and CSP, a flexible and extensible platform is required to support the agent management. For instance, if a VC agent is launched into the platform, it will proactively seek out the services of other agents it requires to fulfill the voltage constraints using the agent and service discovery provisions of the agent management system (AMS) and directory facilitator (DF). Agents can be easily added or

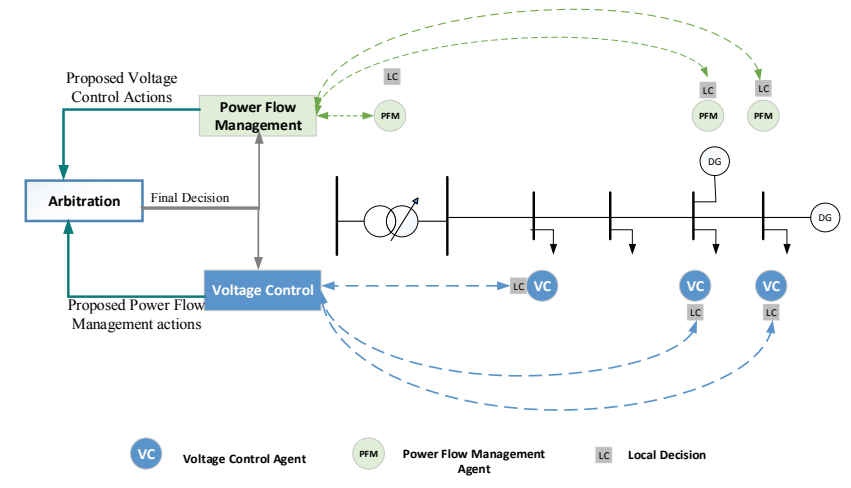

Fig. 7. Hybrid MAS architecture with arbitration 
removed to actively support the control or management functionalities.

\section{Case Study}

Comparing with the existing distribution management system, the proposed ANM system has tighter limits for voltage and thermal, therefore it will act as soon as the voltage/thermal level reach the limit and control the system quickly, i.e. the voltage limit has been set to $3 \%$ for this case study.

\subsection{Case study network}

The case study network is a section of $11 \mathrm{kV}$ radial system with two DG units (Fig. 8) and three transformers. The DG units are non-firm generators which allows power curtailment. Also the DG1 connects on the network later than DG2 which makes the DG2 curtailment more expensive than DG1 based on the LIFO policy widely implemented by the DNOs. The network consists of two feeders, controllable resources include OLTCs, DG active/reactive power set point control and load shedding. The details of the case study network are presented in Table 2 .

\subsection{Testing environment}

Physically, the testing environment includes two set of

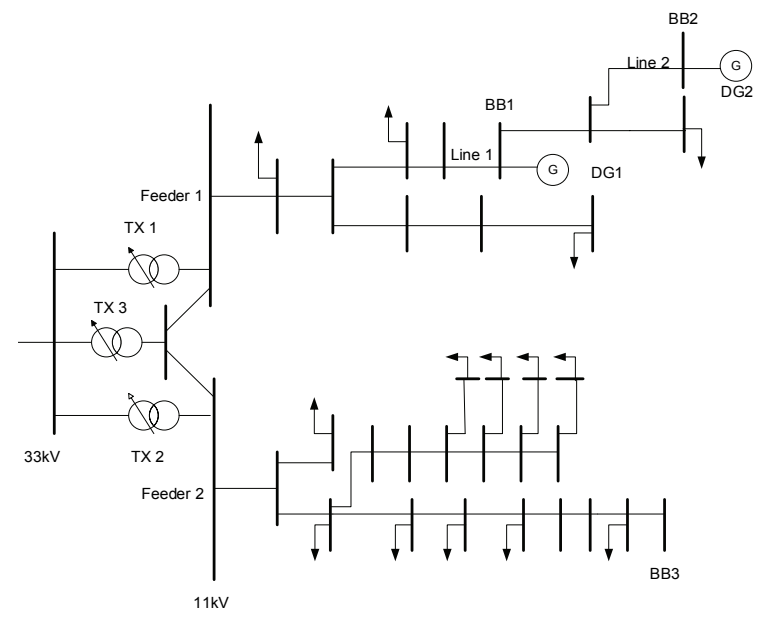

Fig. 8. Case study network

Table 2. Details of the equipment on the case study network

\begin{tabular}{|c|c|c|}
\hline Equipment & Operating range & Details \\
\hline DG1 & $\begin{array}{c}{\left[\begin{array}{ll}0 & 1.9\end{array}\right] \mathrm{MW}} \\
{\left[\begin{array}{ll}0 & 0.6\end{array}\right] \mathrm{MVAr}}\end{array}$ & $\begin{array}{c}\text { Landfill gas; } \\
\text { Connected on } 2008\end{array}$ \\
\hline DG2 & $\begin{array}{l}{\left[\begin{array}{ll}0 & 1.6\end{array}\right] \mathrm{MW}} \\
{\left[\begin{array}{ll}0 & 0.8\end{array}\right] \mathrm{MVAr}}\end{array}$ & $\begin{array}{c}\text { Landfill gas; } \\
\text { Connected on } 2007\end{array}$ \\
\hline Loads & $\begin{array}{l}{[30 \%, 100 \%] \text { of }} \\
\text { peak load values }\end{array}$ & \\
\hline Transformers 1,2 and 3 & {$[-10 \%, 10 \%]$} & Tap step $=1 \%$ \\
\hline
\end{tabular}

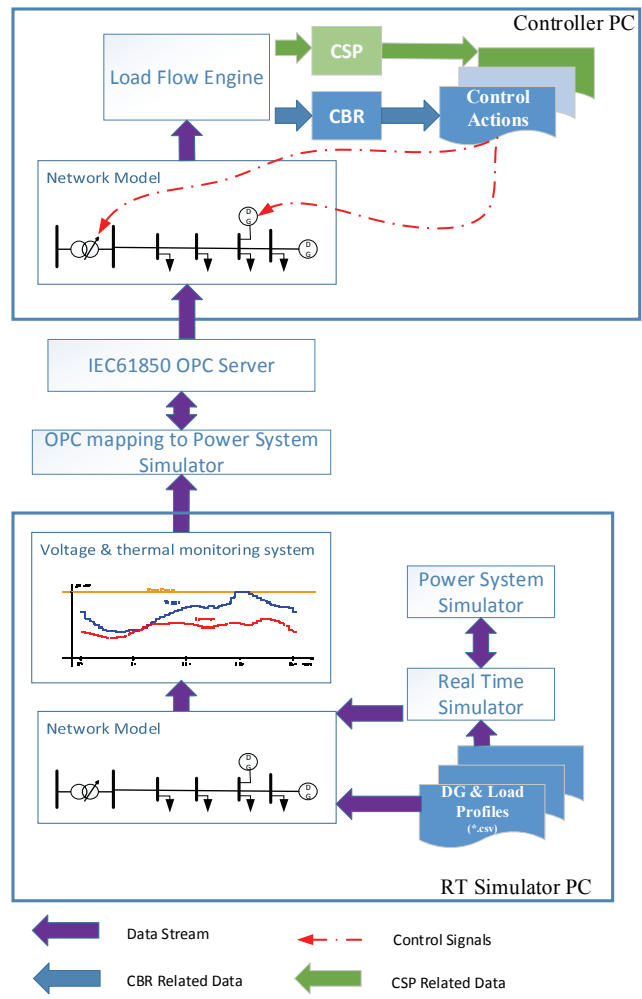

Fig. 9. Schematic diagram of the testing environment

PCs (Fig. 9), one acts as RT simulator and the other one uses for controller. For the RT simulator, the test network parameters (loads, generators, breakers, tap-changers etc.) are changed in real time according to either predefined profiles or any controller actions. The load flow analysis runs every time a change is detected in this quasi-stationary model of the test network. For the controller PC, VC and PFM schemes run with a load flow engine and a network model to represent the close loop control on the network. It reacts to any change of the control data and applies the change to the simulated network.

Using the communication protocols (OPC container and IEC61850 standard), the control algorithms read and write to the communication gateway in the same way they would do in the real power network. The RT simulator runs the pre-defined load and generator profiles and feeds the data to the controller. The RT simulator is implemented in $\mathrm{C}++$ and uses the embedded Python interface to IPSA+ for the load-flow engine and manipulation of the network parameters [18]

For real implementations, the practical management system will be physically located in the substation which can manage several feeders.

\subsection{Flow Chart}

Utilizing the bespoke testing environment, the overall system working flow chart is briefly described in Fig.10. It can be explained in the following steps: 
(1) The system monitoring agent observe the system voltage and thermal levels to identity any excursions. The breach of the thermal limits could damage components or reduce the life expectancy in a short period of time, hence the system checks the thermal constraint before voltage.

(2) Once an excursion has been detected, the VC or PFM agent will be requested to provide a set of ranked solutions.

(3) All proposed solutions will be verified through an online verification agent (run load flow with updated parameters) to confirm that the solutions can solve the excursion detected and will not breach other limits.

(4) The successful verified solutions are passed to check the other constraint. If the solutions satisfy both constraints, voltage and thermal, they are ready for implementation. Then, the artificial costs are added to the proposed solutions and an overall score is calculated according to Eq. (13) for each recommended solutions to rearrange the rank of preference. And the solution with highest overall score is claimed as the solution for close loop implementation.

(5) Once the solution failed in the other constraint, the system will run the other function, CBR or CSP to make sure both constraints are satisfied.

(6) In order to avoid endless loop, an arbitration enquiry ( $m$ or $n$ ) will be sent to the operating center. When the sum number of enquires ( $\mathrm{M}$ or $\mathrm{N}$ ) reach the maximum limits the system will stop the loop and send both control actions to the control center for judgment.

\subsection{Testing scenarios}

Four set of testing scenarios have been carried out in

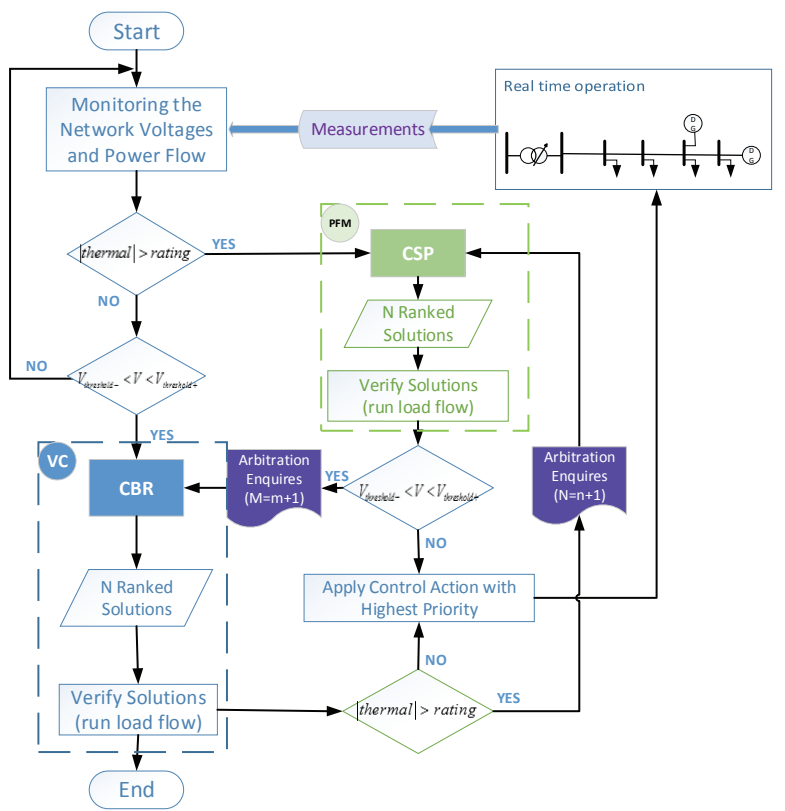

Fig. 10. Flow chart of the proposed integrated ANM system this paper, including independent voltage excursion and thermal excursion scenarios, composed voltage and thermal excursions and fault tolerant testing with data loss during the operation.

\section{Test 1: voltage excursion only}

In order to verify the active $\mathrm{VC}$ strategies, the voltage limits has been de-rated as 3\%. Introducing predefined load and generation profiles, via the sequential steady state testing environment, allows the emulation of voltage excursions on DG2 connected busbar BB2 (Fig. 11(a)). The proposed CBR system collect all the related information and wrap the excursion scenario as a case, the retrieved solution comprises DG unit set point control first and flowed by OLTCs (Fig. 11(b)-11(c)). Generally the DG ramp rate reacts quick than OLTCs, whilst the OLTCs solve the voltage rise issue more efficiently without bother the DG owner's revenue. Furthermore, according to the LIFO policy, DG2 curtailment is more expensive than DG1, therefore DG1 constrained more power than DG2.

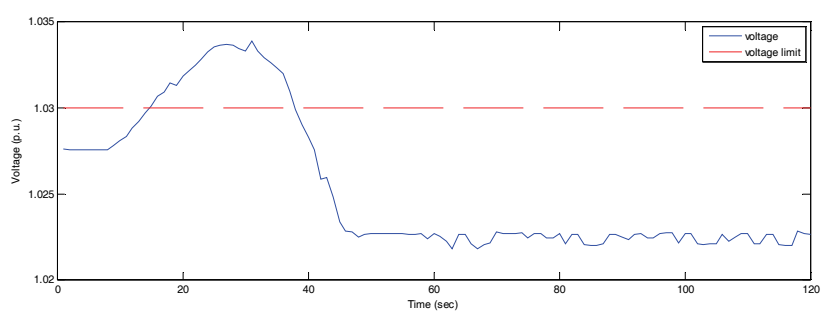

(a)

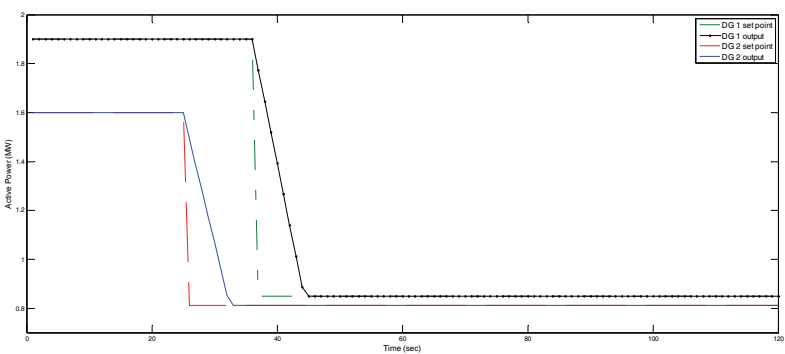

(b)

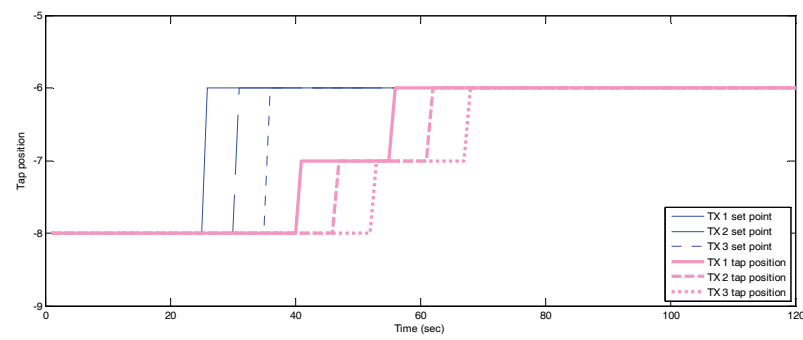

(c)

Fig. 11. (a) Voltage profile with an excursion on DG2 connection point; (b) DG1 \& DG2 P set point change and active power produced; (c) Transformer set point and associate tap position 
Following the control actions, the voltage level back within the statutory limits and the thermal restrictions are not being breached. The retrieved 5 cases with corresponding control actions and their associated artificial cost is illustrated in Table A in the Appendix, the third control solution has been chosen for implementation.

\section{Test 2: Thermal excursion only}

During normal operating conditions, the cables and transformers can have up to $50 \%$ of their capacity redundant in order to be able to provide support during system reconfiguration in the event of an outage on the network [15] Hence, the ratings of the cables and transformers are being de-rated during the tests which allows the emulation of thermal excursions on the location of thermally vulnerable components within the distribution network.

The domain is set to be $\{0,0.2,0.5,0.8,1\}$ for the DG units which indicates that the DG power can be curtailed in a certain amount. Once a thermal excursion has been detected, the CSP algorithm searches for appropriate solutions. During the tests, LIFO policy and the availability of controllable loads also acts as constraints. Fig. 12 demonstrates the simulation results with DG1 and DG2 power curtailment in line with the connection order to alleviate the line overload. The DG1 is being trimmed $80 \%$ of the overall active power output, with $20 \%$ power reduction on DG 2 within a shorter period of time.

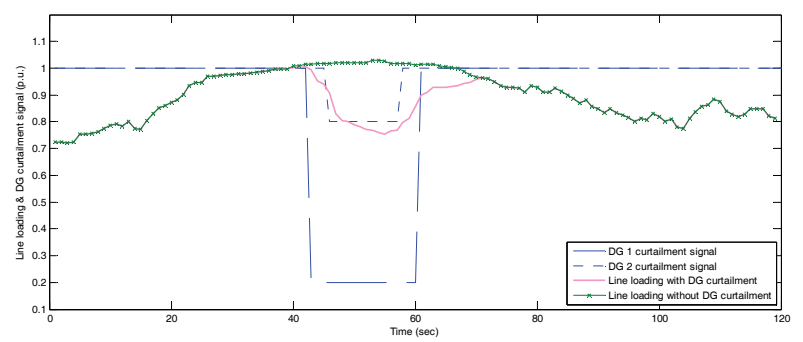

Fig. 12. DG control signals and line loading with/without DG curtailment

\section{Test 3: Composed thermal and voltage excursions}

Under normal operating condition, the following two events can possibly cause composed thermal and voltage excursions:

(1) When a thermal excursion occurs on the network, DG units have been ramped down, consequently, the voltage level on feeder 2 (feeder without DG) may drop below the limit;

(2) On a weak feeder without DG units, for instance feeder 2, the voltage level on busbars drop below the voltage limit, taps may tap down, therefore the thermal limits are being breached on other feeders near the DG connection point.
For the first event, when the thermal excursion has been solved, normally the DG units are required to ramp up to achieve the revenue of the DG, therefore the voltage on feeder 2 can normally back within the limits in a short period of time without additional control.

For the second event, when the thermal excursions are being detected, an arbitration enquiry is being sent to PFM agent to solve the thermal issue, the DG units are being ramped down, and the voltage on busbar would experience voltage under limit issue again, an arbitration enquiry will be sent to $\mathrm{VC}$ agent, the $\mathrm{VC}$ agent has noticed the preceding event. The self-learning strategy notify the CBR system to avoid the transformer operation due to the previous conflict. Fig. 13 (a)-(c) explain the whole process of the composed thermal and voltage excursions. As shown in the figures, when the first voltage excursion has been detected, the CBR issues OLTCs control signals, therefore the voltage level is being improved. Nevertheless, the

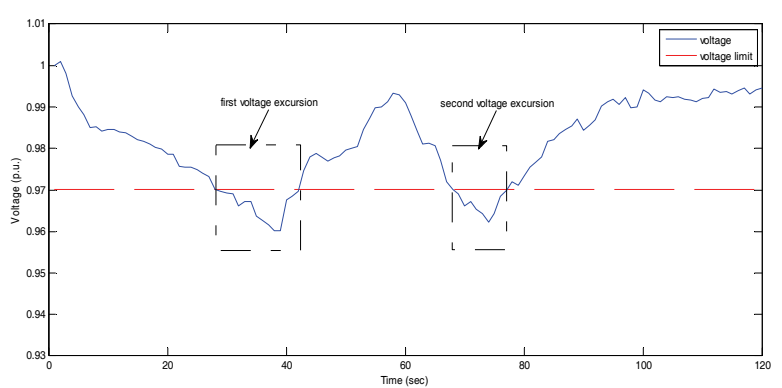

(a)

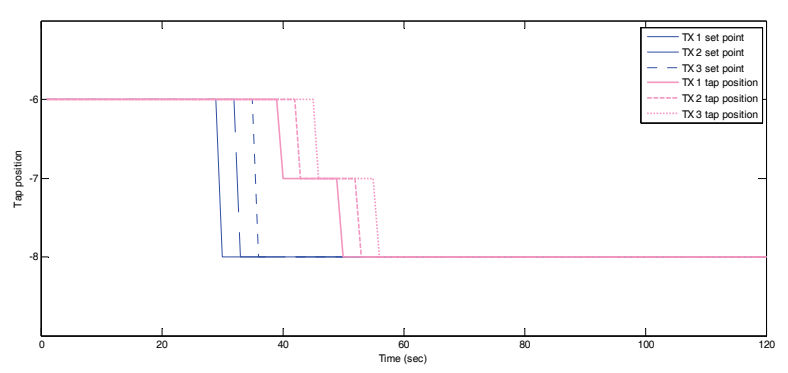

(b)

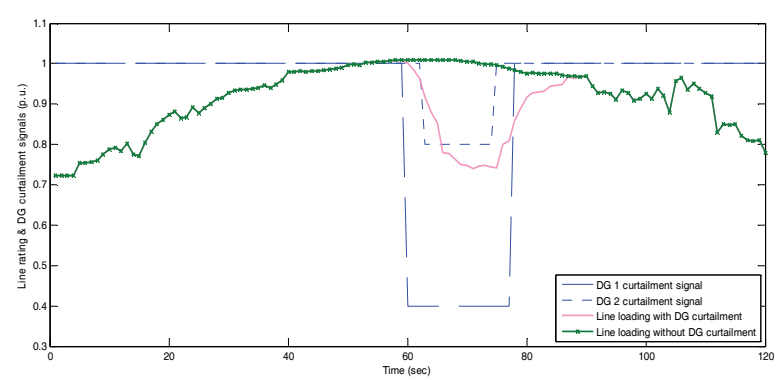

(c)

Fig. 13. (a) Voltage profile on busbar BB3 with two excursions; (b) Transformer set point and associate tap position; (c) DG control signals and line loading with/without DG curtailment 
thermal limit on "Line2" is being breached, thus the CSP algorithm constraints the power output of the DG units. During the operation of DG curtailments, the voltage level on BB3 drops below the limit until the DG power reductions have been released.

\section{Test 4: Voltage control with data corruption}

Incomplete information, data loss and failure of communication have become obstructions in ANM scheme implementation. The test carried out here is to simulate a load change which leads to an over-voltage scenario, but with the added complexity of partial data loss. Fig. 14(a)(b) illustrates the CBR VC algorithm with data loss on busbar BB2. As long as a data loss occurred during the simulation, the RT simulator normally keeps the data unchanged until new measurement feeds in. The results of the real time test shows that the algorithm is capable of detecting and eliminating the over-voltage issue by adjusting the tap changers even in the presence of intermittent data loss.

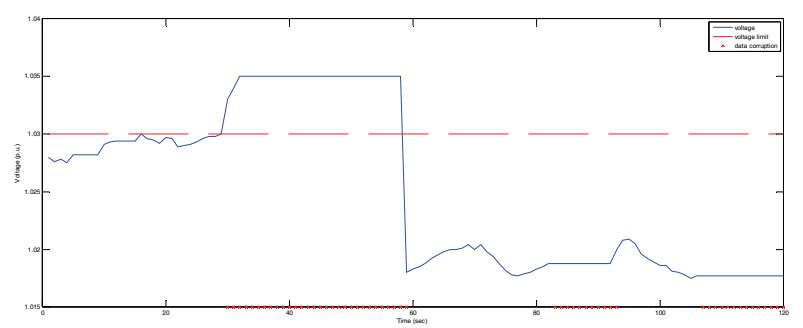

(a)

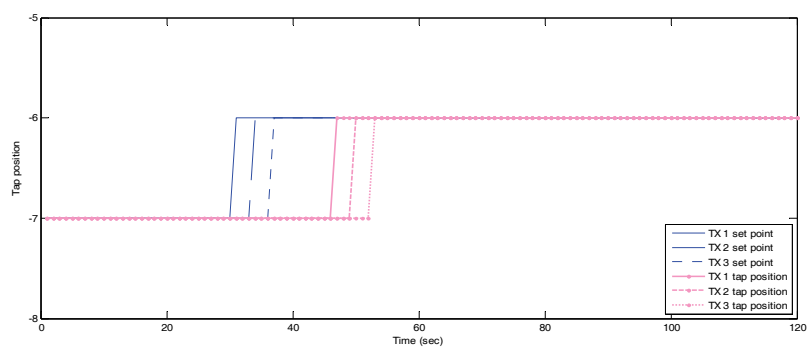

(b)

Fig. 14. (a) Voltage profile on busbar BB2 with data corruption; (b) Transformer set point and associate tap position

\section{Concluding Remarks}

It is anticipated that a significant levels of DG will connected on the distribution networks in the coming years. Taking an ANM schemes in controlling the voltage and thermal levels within statutory limits by utilizing existing network capacities and infrastructure can consequently avoid unnecessary network reinforcement, reduce losses and increase the power sourced from the renewables. The aim of this research is to investigate the efficiency of active solutions utilizing the proposed CBR and the CSP algorithms, in particular, to address the role of collaborative approach. The CBR system which is characterized by self-learning retrieves VC solutions with knowledge of previous implementation experiences. On the other hand, the CSP algorithm searches for available solutions by regulating the real power output from connected DG units and controllable loads. Both strategies provide a set of solutions just in case the selected solution fail to resolve the voltage/thermal breach a fall back situation exists.

A novel testing environment is developed to carry out a series of testing including independent and composed voltage/thermal control, as well as data corruptions to verify the accuracy, efficiency and fault tolerance of the proposed approach. The testing results and computation speed have demonstrated that the proposed collaborative approach has the potential to be embedded in an online control system with a view to managing the output of multiple DG schemes, based on real-time knowledge of the voltage/thermal and electrical status of the distribution network.

Further research is also required on the analysis of the operation cost of each control solutions, incentive strategies for each participated DG and controllable loads, as well as the additional control methods. Arbitration strategy which employs intelligent algorithm such as game theory have promising potential in solving confliction between control solutions.

\section{Acknowledgements}

This work was supported by the National Key Technology R\&D Program of China (2013BAA01B03). and the National Nature Science Foundation of China(51307118, 51261130473) and the "131" Talents Program of Tianjin.

\section{References}

[1] Tao Xu and Phil Taylor, "Voltage control techniques for electrical distribution networks including distributed generation", in Proceedings of 17th IFAC world congress, Seoul, Korea, July 2008.

[2] Sam Jupe and Phil Taylor, "Strategies for the control of multiple Distributed Generation", in Proceedings of 20th International Conference and Exhibition on Electricity Distribution (CIRED), Prague, Czech Republic, June 2009.

[3] Panagis N. Vovos, Aristides E. Kirprakis, Robin Wallace, "Centralised and distributed voltage control: impact on distributed generation penetration", IEEE 
Trans. Power Systems, vol. 22, No. 1, pp. 476-482, Jan 2007.

[4] Gram Ault, Jim McDonaid, "Fundamental research challenges for active management of distribution networks with high levels of renewable generation", in Proceedings of Universities Power Engineering Conference, Manchester, UK, March 2004.

[5] Dave Roberts, "Network management systems for active distribution networks: a feasibility study", DTI Distributed Generation Programme, 2004.

[6] Phuong Nguyen and Will Kling, "Distributed state estimation for multi-agent based active distribution networks", in Proceedings of IEEE PES General Meeting, Minneapolis, USA, July 2010.

[7] Scottish and Southern Energy, The Orkney smart grid, http://www.ssepd.co.uk/OrkneySmartGrid/Knowledge Sharing/Events/OrkneyEventMaterial/, January 2015.

[8] Mark Collins, Richard Silversides and Tim Green, "Control and coordination of a distribution network via decentralised decision making", in Proceedings of IEEE PES General Meeting, Minneapolis, USA, 2013.

[9] Phil Taylor, Tao Xu, Neal Wade, Milan Prodanovic, Richard Silversides, Tim Green, Euan Davidson, and Stephen McArthur, "Distributed voltage control in AuRA-NMS", in Proceedings of IEEE PES General Meeting, Minneapolis, USA, July 2010.

[10] Phuong Nguyen and Will Kling, "Coordination of voltage regulation in active networks", in Proceedings of IEEE PES Transmission and Distribution Conference and Exposition, Chicago, USA, Apr 2008.

[11] Ali Ahmadi and Tim Green, "Optimal power flow for autonomous regional active network management system", in Proceedings of IEEE PES General Meeting, Minneapolis, USA, 2009.

[12] Sam Jupe and Phil Taylor, "Distributed generation output control for network power flow management", IET Renewable Power Generation, vol. 3, No. 4, pp. 371-386, June 2009.

[13] Neal. Wade, Phil Taylor, Peter Lang, "Evaluating the benefits of an electrical energy storage system in a future smart grid", Energy Policy, vol. 38, No. 11, pp.
7180-7188, June 2011.

[14] Tao Xu, Neal Wade, Euan Davidson, Phil Taylor, Stephen McArthur, "Case-Based Reasoning for Coordinated Voltage Control on Distribution Networks", Electric Power Systems Research, vol. 81, No. 12, pp. 2088-2098, Dec 2011.

[15] Mike Dolan, Euan Davidson, "Reducing distributed generator curtailment through active power flow management", IEEE Trans. On smart grid, vol. 5, No. 1, pp. 149-157, Jan 2014.

[16] Edward Tsing, "Foundation of Constraint Satisfaction". USA: Academic Press, 1993, p. 1-10.

[17] Krzysztof Apt, "Principles of Constraint Programming”, UK: Cambridge Univ. Press, 2003, p. 3440.

[18] IPSA+ power website, http://www.ipsa-power.com/, January 2015.

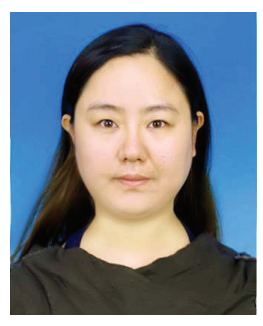

Tao Xu She received her Ph.D. degree in the School of Engineering, Plymouth University, UK. She is currently a lecture in the Key Laboratory of Smart Grid of Ministry of Education, Tianjin University. She carries out research which concentrates on the challenges associated with the widespread integration and control of distributed/ renewable generation in electrical networks.

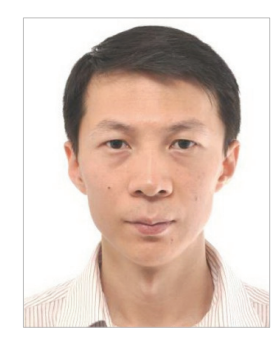

Lingxu Guo He received his B. Eng. and M.Eng degree in the School of Electrical Engineering and Automation, Tianjin University, China. He is now a senior engineer in the State Grid Tianjin Electric Power Co. His research interests are the operation and control of electrical power system.

\section{Appendix}

Table A. Ranked control solutions retrieved from the CBR system

\begin{tabular}{c|c|l|c|c|c|c|c}
\hline No. & $\begin{array}{c}\text { Type of } \\
\text { control }\end{array}$ & \multicolumn{1}{|c|}{ Control actions } & $\begin{array}{c}\text { Overall } \\
\text { similarity }\end{array}$ & $\begin{array}{c}\text { Artificial cost } \\
\text { of voltage }\end{array}$ & $\begin{array}{c}\text { Artificial cost } \\
\text { of power } \\
\text { curtailment }\end{array}$ & $\begin{array}{c}\text { Verification result } \\
\text { (pass=1; fail=0) }\end{array}$ & $\begin{array}{c}\text { Overall } \\
\text { score }\end{array}$ \\
\hline 1 & OLTC & Transformers 1, 2 and 3 tap position=-5\% & 0.925 & 2.9860 & 0 & 1 & -0.568 \\
\hline 2 & P set point & DG 1 P=0.50 MW; DG 2 P=0.70 MW & 0.913 & 0.2621 & 1.650 & 1 & -0.043 \\
\hline 3 & $\begin{array}{c}\text { P set point } \\
\text { +OLTC }\end{array}$ & $\begin{array}{l}\text { DG 1 P=0.80 MW; DG 2 P=0.83 MW; } \\
\text { Transformers 1, 2 and 3 tap position=-6 }\end{array}$ & 0.913 & 0.4644 & 1.353 & 1 & 0.0043 \\
\hline 4 & $\begin{array}{c}\text { PF set point } \\
\text { +OLTC }\end{array}$ & $\begin{array}{l}\text { DG 1 P=0.80 MW; DG 1 Q }=0.2 \text { Mvar; } \\
\text { DG 2 P=0.90 MW; DG 2 Q=0.5 Mvar; } \\
\text { Transformers 1, 2 and 3 tap position=-6 }\end{array}$ & 0.893 & 1.0742 & 1.410 & 0 & 0 \\
\hline 5 & OLTC & Transformers 1, 2 and 3 tap position=-4\% & 0.867 & 1.9738 & 0 & & 0 \\
\hline
\end{tabular}




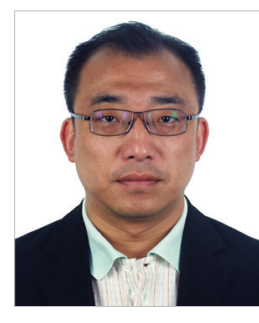

Wei Wei He completed his B. Eng., M.Eng. and Ph.D. in the School of Electrical Engineering and Automation, Tianjin University, China. He is now an associate professor in the Key Laboratory of Smart Grid of Ministry of Education, Tianjin University. His research interests are the power system analysis and planning.

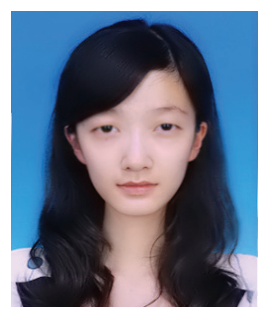

Xiaoxue Wang She received her B. Eng. from the School of Electrical Engineering and Automation, Tianjin University, China. She is currently a Ph.D. student in the School of Electrical Engineering and Automation, Tianjin University, China. Her research interests are the active distribution system voltage control and distributed intelligence on the power system.

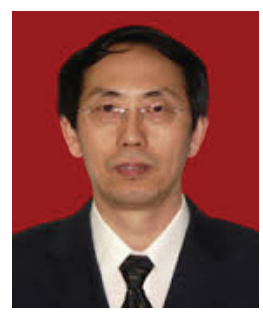

Chengshan Wang He received his B. Eng., M.Eng. and Ph.D. degree in the School of Electrical Engineering and Automation, Tianjin University, China. $\mathrm{He}$ is a professor and the director of the Key Laboratory of Smart Grid of Ministry of Education, Tianjin University. His research interests are system security and stability, distribution system analysis and planning and distributed generation system modeling and control.

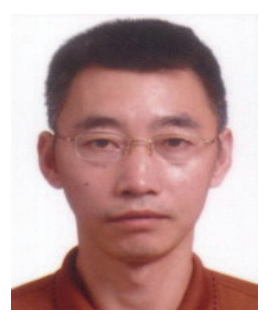

Jun Lin $\mathrm{He}$ is currently a senior engineer in Hainan Electric Power Research Institute.

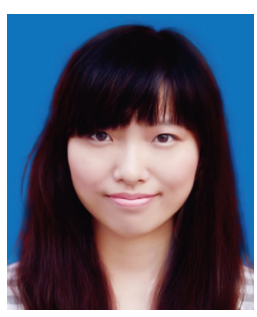

Tianchu Li received her B. Eng. Degree from the North China Electric Power University. She is currently an engineer in Hainan Electric Power Research Institute. 